\title{
Achados oftalmológicos em pacientes que receberam transplante cardíaco
}

\author{
Ophthalmologic findings in cardiac transplant recipients
}

\author{
Cecília Sales Pires ${ }^{1}$ \\ Maria Cecília de Aguiar Remígio ${ }^{2}$ \\ Maria Inês Remígio de Ag'uiar $^{3}$ \\ Deuzeny Tenório ${ }^{4}$ \\ Carlos R. Moraes 5 \\ Hellman Dantas de Olinda Cavalcanti ${ }^{6}$
}

\begin{tabular}{|l|}
\hline RESUMO \\
\hline Objetivo: Avaliar os achados oculares, pelo exame oftalmológico, em \\
indivíduos que receberam transplante cardíaco, buscando especialmente \\
investigar possíveis alterações na camada de fibras nervosas da retina com \\
polarímetro de varredura a laser. Métodos: Foram estudados 15 indivíduos \\
que receberam transplante cardíaco no período de setembro de 2003 a \\
julho de 2004. Todos foram submetidos a exame que constava de acuidade \\
visual para longe (AVL), biomicroscopia, tonometria e fundoscopia. Onze \\
pacientes foram submetidos ao analisador de fibras nervosas GDx. Doze \\
eram do sexo masculino e a média da idade foi de $55,0 \pm 13,5$ anos. O tempo \\
decorrido desde o transplante variou de 3 a 74 meses, com média de 29,7 \\
$\pm 20,8$ meses. Resultados: A AVL com melhor correção foi igual ou \\
melhor do que 20/40 em todos os pacientes. Em um deles observou-se a \\
presença de catarata subcapsular posterior; em outro, nubéculas na córnea \\
secundárias a quadro de herpes zoster. À fundoscopia pôde-se observar \\
lesão cicatrizada sugestiva de retinocoroidite em um paciente. As alterações \\
observadas à biomicroscopia e à fundoscopia eram esperadas devido à \\
imunossupressão subseqüente ao transplante. Ao GDx observou-se perda \\
de fibras da camada de fibras nervosas da retina superior em 12 dos 22 \\
olhos avaliados. Conclusão: Os resultados apóiam a suposição de que \\
antes ou durante o transplante cardíaco tenha havido diminuição no \\
aporte de oxigênio à circulação retiniana, levando a perda parcial de \\
fibras da retina.
\end{tabular}

Descritores: Transplante de coração; Manifestações oculares/etiologia; Fibras nervosas; Técnicas de diagnóstico oftalmológico

\section{INTRODUÇÃOO}

O transplante cardíaco é uma modalidade de tratamento cirúrgico bem estabelecida para doentes com cardiopatia em estado terminal depois de esgotadas todas as possibilidades de terapêutica clínica e cirúrgica convencional. A maioria dos candidatos se encontra na classe IV e alguns poucos na classe III da classificação da New York Heart Association. Sem tratamento a qualidade de vida é má e a sobrevida vai de 3 a 12 meses $^{(1-2)}$. As indicações mais comuns são a cadiopatia isquêmica com 50,6\%; as cardiomiopatias com 40,9\%; outros tipos de doença cardíaca com 5,3\% e o retransplante com $3,2 \%{ }^{(1)}$.

Complicações oculares associadas a transplante de órgãos já foram bem documentadas em pacientes que receberam transplantes renais. São poucos os estudos em transplantados cardíacos, havendo porém relatos de casos de linfoma intra-ocular, descolamento de retina, retinite por citomegalovírus e oclusão da veia central da retina após transplante cardíaco ${ }^{(3-6)}$. 
O uso de drogas imunossupressoras como azatioprina, ciclosporina A e prednisona tendo como objetivo diminuir a intensidade da resposta imunológica global, pode ocasionar complicações oculares importantes ${ }^{(7)}$.

Em um estudo com análise histopatológica de cérebro de 262 pacientes submetidos a transplante cardíaco, a "bypass" coronário ou troca de válvula cardíaca, encontraram-se alterações circulatórias como macro e microhemorragias, infartos e lesões por isquemia, entre outros ${ }^{(8)}$. No entanto, não foram encontrados relatos sobre essas alterações na microcirculação da retina.

Este estudo, realizado em um grupo de pacientes submetidos a transplante cardíaco, consistiu em exame oftalmológico incluindo busca de possíveis alterações da camada de fibras nervosas da retina com polarímetro de varredura a laser.

\section{MÉTODOS}

Foram estudados 15 pacientes submetidos a transplante cardíaco encaminhados pelo Instituto do Coração do Hospital Português à Fundação Altino Ventura (FAV) no período de setembro de 2003 a julho de 2004. Foram encaminhados para exame apenas os pacientes que utilizavam ciclosporina $\mathrm{A}$, micofenolato e prednisona como tratamento imunossupressor.

Doze pacientes eram do sexo masculino. A idade variou de 23 a 75 anos, com média de 55,0 $\pm 13,5$ anos. O grupo de idades entre 50 e 65 anos representou $65 \%$ do total. O tempo decorrido desde o transplante cardíaco variou de 3 a 74 meses, com média de 29,7 $\pm 20,8$ meses. Doze dos pacientes apresentavam hipertensão arterial sistêmica e 5 desses pacientes apresentavam diabetes mellitus associada.

Todos os pacientes foram avaliados pelo mesmo examinador nos ambulatórios da FAV. Foram submetidos a exame oftalmológico que constava de acuidade visual para longe (AVL) com a melhor correção pela tabela E de Snellen, biomicroscopia, tonometria de aplanação com tonômetro de Goldmann e fundoscopia indireta com lente de Volk 90. Onze pacientes (22 olhos) foram submetidos ao exame com o analisador de fibras nervosas GDx. Dois pacientes foram excluídos por suspeita de glaucoma e dois por não comparecerem na data prevista.

Os resultados foram expressos por suas freqüências, médias e respectivos desvios-padrão.

O projeto foi aprovado pela Comissão de Ética da FAV. Os pacientes que participaram do estudo assinaram um termo de consentimento livre e esclarecido.

\section{RESULTADOS}

A AVL com melhor correção foi melhor ou igual a 20/40 em todos os pacientes, tendo um deles apresentado este valor em ambos os olhos. Outro apresentou 20/40 em um olho e 20/20 no outro.
Observou-se à biomicroscopia presença de catarata subcapsular posterior (SCP) em um paciente (Quadro 1).

Todas as pressões intra-oculares (PIO) estiveram dentro dos limites da normalidade, variando de 8 a $19 \mathrm{mmHg}$ sendo a média de $13,3 \pm 2,5$ no olho direito e de $13,5 \pm 1,7$ no olho esquerdo.

A presença de rarefação difusa do epitélio pigmentar da retina (EPR) foi a alteração mais freqüente no exame do fundo de olho (FO), presente em $10(33,3 \%)$ olhos. Na periferia nasal do OD de um paciente observou-se lesão cicatrizada de um diâmetro de disco (DD) de tamanho, sugestiva de retinocoroidite, secundária a uveíte posterior. Alterações da escavação papilar (EP) sugestivas de glaucoma foram vistas à fundoscopia em ambos os olhos de dois pacientes (Quadro 2).

Ao GDx evidenciou-se em 12 dos 22 olhos analisados, perda de fibras da camada de fibras nervosas da retina superior. Em um olho, observou-se perda temporal associada à perda superior destas fibras nervosas e em outro, observou-se perda difusa da camada de fibras nervosas. Em 10 olhos o exame foi normal, com todos os parâmetros dentro dos limites da normalidade. O número, valor preditivo de glaucoma mais importante deste exame, foi normal em 19 olhos e limítrofe em três (Tabela 1).

\section{DISCUSSÃO}

A presença de catarata subcapsular posterior é o achado mais comum após o uso crônico de corticosteróide em transplantados $^{(9)}$. Neste estudo observou-se proporção de $6,7 \%$, inferior à da literatura (12,5 a 60,0\%), o que é provavelmente explicado pelo pequeno número de casos, dos quais três pacientes submetidos a facectomia. O tempo decorrido desde o transplante, em média inferior a três anos, talvez não seja suficiente para o desenvolvimento de alterações cristalinianas, já que só após três anos do uso de corticóides elas começam a se manifestar ${ }^{(7)}$

Outro achado à biomicroscopia, relacionado talvez à imunossupressão a que se submeteram os pacientes, foi a presença de nubéculas na córnea do olho direito de um deles, conseqüente a herpes zoster ocular.

Nesta investigação confirmaram-se os achados de outros estudos quanto à acuidade visual após transplante cardíaco. Embora não se tenha informação da AVL prévia ao transplante, não houve grande piora da mesma após o transplante, pois a AVL foi igual ou superior a 20/40 em todos os pacientes ${ }^{(7)}$.

A lesão cicatrizada com um DD de tamanho, sugestiva de retinocoroidite, pode ter sido causada por infecção oportunista, freqüentes em pacientes imunossuprimidos. Ressaltese que as infecções oportunistas são comuns como causa de afecções pós transplante ${ }^{(2,7,9)}$.

Não se observaram outras alterações fundoscópicas associadas ao transplante ou à imunossupressão que lhe seguiu.

Não se observou PIO elevada, o que sugere ausência de relação entre transplante cardíaco e aumento de pressão que 
possa levar ao desenvolvimento de glaucoma. Sabe-se, contudo, que o uso prolongado de corticosteróides pode levar ao aumento da PIO. O curto período de seu uso desde o transplante impossibilita conclusões a esse respeito.

Constatou-se perda parcial da camada de fibras nervosas da retina superior em $54,6 \%$ dos olhos, o que pode sugerir uma diminuição no aporte de oxigênio para a circulação da retina durante o transplante.

Alterações circulatórias durante cirurgias cardíacas já foram relacionadas a lesões cerebrais em outros estudos ${ }^{(8,10)}$. Todavia, não se pode descartar a possibilidade da perda de fibras nervosas ter ocorrido no período pré-transplante, pois as doenças que levam a esse procedimento são hipoxemiantes. Não existem relatos relacionando alterações circulatórias associadas a transplantes cardíacos com danos na camada de fibras nervosas da retina. No entanto, perda de células nervosas da retina in vitro por isquemia já foi descrito ${ }^{(11)}$.

Sugere-se a realização de outros estudos com maior número de pacientes e avaliação pela análise de fibras nervosas da retina antes e depois do transplante cardíaco. Também se poderia tentar determinar o fluxo de sangue ocular durante a cirurgia a fim de investigar se ocorre diminuição no aporte de oxigênio ao olho.

\section{CONCLUSÃO}

Os resultados apóiam a suposição de que antes ou durante o transplante cardíaco tenha havido uma diminuição no aporte

\begin{tabular}{|c|c|c|c|c|}
\hline \multicolumn{5}{|c|}{ Quadro 1. Achados à biomicroscopia } \\
\hline Paciente № & Tempo desde & o transplante (meses) & Biomicroscopia do olho direito & Biomicroscopia do olho esquerdo \\
\hline 1 & & 12 & Sem alterações aparentes & Sem alterações aparentes \\
\hline 2 & & 5 & Pinguécula nasal e temporal & Pinguécula nasal e temporal \\
\hline 3 & & 3 & Triquíase / pseudofacia & Pseudofacia \\
\hline 4 & & 24 & Pseudofacia & Pseudofacia \\
\hline 5 & & 23 & Meibomite & Meibomite \\
\hline 6 & & 41 & SCP e cortical anterior na periferia & SCP e cortical anterior na periferia \\
\hline 7 & & 33 & Chagrém na córnea & Chagrém na córnea \\
\hline 8 & & 24 & Pinguécula nasal e temporal / NS +/4+ & Pinguécula nasal e temporal / NS +/4+ \\
\hline 9 & & 42 & Sem alterações aparentes & Sem alterações aparentes \\
\hline 10 & & 53 & Sem alterações aparentes & Sem alterações aparentes \\
\hline 11 & & 30 & Pterígio nasal / Chagrém na córnea & Chagrém \\
\hline 12 & & 9 & Pinguécula nasal e temporal & Pinguécula nasal e temporal \\
\hline 13 & & 14 & Nubéculas secundárias a quadro de $\mathrm{HZ}$ & Nódulo de Salzman \\
\hline 14 & & 59 & $\mathrm{NS}+/ 4+$ & NS $+/ 4+$ \\
\hline 15 & & 74 & Pseudofacia & Pseudofacia \\
\hline
\end{tabular}

\begin{tabular}{|c|c|c|c|}
\hline \multicolumn{4}{|c|}{ Quadro 2. Achados à fundoscopia } \\
\hline Paciente № & Idade (anos) & Fundoscopia do olho direito & Fundoscopia do olho esquerdo \\
\hline 1 & 23 & Sem alterações aparentes & Sem alterações aparentes \\
\hline 2 & 39 & Lesão de 1 DD cicatrizada na periferia nasal & Sem alterações aparentes \\
\hline 3 & 64 & NO hipocorado / rarefação do EPR & NO hipocorado / rarefação do EPR \\
\hline 4 & 60 & $\begin{array}{l}\text { Exsudatos duros na região temporal, } \\
\text { não atinge mácula }\end{array}$ & Sem alterações aparentes \\
\hline 5 & 54 & Sem alterações aparentes & Sem alterações aparentes \\
\hline 6 & 56 & Retinopatia hipertensiva grau I & Retinopatia hipertensiva grau I \\
\hline 7 & 56 & Rarefação do EPR & Rarefação do EPR \\
\hline 8 & 59 & Sem alterações aparentes & Sem alterações aparentes \\
\hline 9 & 65 & Rarefação do EPR & Rarefação do EPR \\
\hline 10 & 49 & Sem alterações aparentes & Sem alterações aparentes \\
\hline 11 & 65 & EP 0,5 vertical por 0,4 horizontal & EP 0,7 vertical por 0,6 horizontal \\
\hline 12 & 37 & Sem alterações aparentes & Sem alterações aparentes \\
\hline 13 & 59 & Rarefação do EPR & Rarefação do EPR \\
\hline 14 & 68 & EP 0,5 vertical por 0,4 horizontal & $\begin{array}{l}\text { EP } 0,6 \text { vertical por } 0,5 \text { horizontal / lesão de } 2 \text { DD } \\
\text { na periferia temporal com hiperpigmentação } \\
\text { do EPR ao redor }\end{array}$ \\
\hline 15 & 75 & $\begin{array}{l}\text { NO hipocorado / rarefação do EPR / } \\
\text { crescente miópica inferior }\end{array}$ & $\begin{array}{l}\text { NO hipocorado / rarefação do EPR / } \\
\text { crescente miópica inferior }\end{array}$ \\
\hline
\end{tabular}




\begin{tabular}{|c|c|c|c|c|c|c|c|c|c|c|c|c|c|}
\hline \multirow{2}{*}{$\begin{array}{l}\text { Paciente № } \\
1\end{array}$} & \multirow{2}{*}{$\begin{array}{l}\text { Olho } \\
\text { OD } \\
\text { OE }\end{array}$} & \multirow{2}{*}{$\begin{array}{c}\text { Sy } \\
1,01 \\
0,97\end{array}$} & SR & IR & $S / N$ & MM & EM & $\mathbf{N}$ & AT & EA & SA & IA & SI \\
\hline & & & $\begin{array}{l}2,39 \\
1,72^{1}\end{array}$ & $\begin{array}{l}2,36 \\
1,77^{1}\end{array}$ & $\begin{array}{l}1,86 \\
2,14\end{array}$ & $\begin{array}{l}1,39 \\
1,20\end{array}$ & $\begin{array}{l}2,14 \\
1,90\end{array}$ & $\begin{array}{l}17 \\
29\end{array}$ & $\begin{array}{l}53 \\
54\end{array}$ & $\begin{array}{l}53^{1} \\
57\end{array}$ & $\begin{array}{l}54^{2} \\
59^{1}\end{array}$ & $\begin{array}{l}66 \\
69\end{array}$ & $\begin{array}{l}0,155^{1} \\
0,165\end{array}$ \\
\hline 2 & $\begin{array}{l}\mathrm{OD} \\
\mathrm{OE}\end{array}$ & $\begin{array}{l}0,78^{1} \\
0,77^{2}\end{array}$ & $\begin{array}{l}2,36 \\
2,17\end{array}$ & $\begin{array}{l}3,04 \\
2,82\end{array}$ & $\begin{array}{l}1,69^{1} \\
1,58\end{array}$ & $\begin{array}{l}2,04 \\
1,82\end{array}$ & $\begin{array}{l}2,77 \\
2,57\end{array}$ & $\begin{array}{l}19 \\
42^{1}\end{array}$ & $\begin{array}{l}47^{1} \\
41^{2}\end{array}$ & $\begin{array}{l}48^{2} \\
45^{2}\end{array}$ & $\begin{array}{l}44^{2} \\
47^{2}\end{array}$ & $\begin{array}{l}63 \\
54^{1}\end{array}$ & $\begin{array}{l}0,121^{2} \\
0,121^{2}\end{array}$ \\
\hline 3 & $\begin{array}{l}\text { OD } \\
\text { OE }\end{array}$ & $\begin{array}{l}0,95 \\
0,95\end{array}$ & $\begin{array}{l}2,08 \\
2,36\end{array}$ & $\begin{array}{l}2,20 \\
2,49\end{array}$ & $\begin{array}{l}2,21 \\
1,99\end{array}$ & $\begin{array}{l}1,34 \\
1,49\end{array}$ & $\begin{array}{l}3,43 \\
2,63\end{array}$ & $\begin{array}{l}10 \\
12\end{array}$ & $\begin{array}{l}66 \\
71\end{array}$ & $\begin{array}{l}67 \\
72\end{array}$ & $\begin{array}{l}68 \\
86\end{array}$ & $\begin{array}{l}85 \\
83\end{array}$ & $\begin{array}{l}0,191 \\
0,236\end{array}$ \\
\hline 4 & $\begin{array}{l}\text { OD } \\
\text { OE }\end{array}$ & $\begin{array}{l}1,04 \\
0,92\end{array}$ & $\begin{array}{l}2,37 \\
1,95\end{array}$ & $\begin{array}{l}2,27 \\
2,12\end{array}$ & $\begin{array}{l}2,18 \\
2,33\end{array}$ & $\begin{array}{l}1,37 \\
1,52\end{array}$ & $\begin{array}{l}2,92 \\
2,41\end{array}$ & $\begin{array}{l}11 \\
12\end{array}$ & $\begin{array}{l}58 \\
53\end{array}$ & $\begin{array}{l}65 \\
56\end{array}$ & $\begin{array}{l}69 \\
59^{1}\end{array}$ & $\begin{array}{l}82 \\
65\end{array}$ & $\begin{array}{l}0,184 \\
0,166\end{array}$ \\
\hline 5 & $\begin{array}{l}\mathrm{OD} \\
\mathrm{OE} \text { * }\end{array}$ & $\begin{array}{l}0,89^{1} \\
0,98\end{array}$ & $\begin{array}{l}2,35 \\
1,99^{1}\end{array}$ & $\begin{array}{l}2,65 \\
2,03\end{array}$ & $\begin{array}{l}1,59^{2} \\
1,66^{1}\end{array}$ & $\begin{array}{l}1,65 \\
1,03^{1}\end{array}$ & $\begin{array}{l}2,65 \\
2,20\end{array}$ & $\begin{array}{l}17 \\
25\end{array}$ & $\begin{array}{l}67 \\
91\end{array}$ & $\begin{array}{l}69 \\
93\end{array}$ & $\begin{array}{l}74 \\
98\end{array}$ & $\begin{array}{r}84 \\
111\end{array}$ & $\begin{array}{l}0,200 \\
0,267\end{array}$ \\
\hline 6 & $\begin{array}{l}\text { OD } \\
\text { OE }\end{array}$ & $\begin{array}{l}0,78^{1} \\
0,91\end{array}$ & $\begin{array}{l}1,56 \\
1,63^{1}\end{array}$ & $\begin{array}{l}2,01 \\
1,79^{1}\end{array}$ & $\begin{array}{l}1,59^{2} \\
1,37^{1}\end{array}$ & $\begin{array}{l}1,05 \\
0,79^{2}\end{array}$ & $\begin{array}{l}2,16 \\
1,87\end{array}$ & $\begin{array}{l}35 \\
49^{1}\end{array}$ & $\begin{array}{l}53 \\
57\end{array}$ & $\begin{array}{l}55 \\
58\end{array}$ & $\begin{array}{l}48^{2} \\
56^{1}\end{array}$ & $\begin{array}{l}67 \\
68\end{array}$ & $\begin{array}{l}0,133^{2} \\
0,153^{1}\end{array}$ \\
\hline 8 & $\begin{array}{l}\text { OD * } \\
\text { OE * }\end{array}$ & $\begin{array}{l}0,80^{2} \\
0,90^{1}\end{array}$ & $\begin{array}{l}1,94 \\
1,70^{2}\end{array}$ & $\begin{array}{l}2,44 \\
1,89\end{array}$ & $\begin{array}{l}1,51^{2} \\
1,66^{1}\end{array}$ & $\begin{array}{l}1,44 \\
0,89^{2}\end{array}$ & $\begin{array}{l}2,90 \\
1,66^{1}\end{array}$ & $\begin{array}{l}23 \\
26\end{array}$ & $\begin{array}{l}71 \\
78\end{array}$ & $\begin{array}{l}73 \\
80\end{array}$ & $\begin{array}{l}75 \\
85\end{array}$ & $\begin{array}{l}92 \\
95\end{array}$ & $\begin{array}{l}0,200 \\
0,230\end{array}$ \\
\hline 9 & $\begin{array}{l}\text { OD } \\
\text { OE }\end{array}$ & $\begin{array}{l}1,06 \\
1,02\end{array}$ & $\begin{array}{l}2,18 \\
2,04\end{array}$ & $\begin{array}{l}2,06 \\
1,99\end{array}$ & $\begin{array}{l}1,78 \\
2,15\end{array}$ & $\begin{array}{l}1,18 \\
1,15\end{array}$ & $\begin{array}{l}3,22 \\
2,67\end{array}$ & $\begin{array}{l}18 \\
23\end{array}$ & $\begin{array}{l}57 \\
52\end{array}$ & $\begin{array}{l}58 \\
56\end{array}$ & $\begin{array}{l}61^{1} \\
64\end{array}$ & $\begin{array}{l}67 \\
65\end{array}$ & $\begin{array}{l}0,160^{1} \\
0,171\end{array}$ \\
\hline 10 & $\begin{array}{l}\text { OD } \\
\text { OE }\end{array}$ & $\begin{array}{l}0,95 \\
1,06\end{array}$ & $\begin{array}{l}2,09 \\
1,95\end{array}$ & $\begin{array}{l}2,21 \\
1,85\end{array}$ & $\begin{array}{l}2,04 \\
1,58\end{array}$ & $\begin{array}{l}1,21 \\
0,95^{1}\end{array}$ & $\begin{array}{l}3,06 \\
2,49\end{array}$ & $\begin{array}{l}16 \\
27\end{array}$ & $\begin{array}{l}51^{1} \\
54\end{array}$ & $\begin{array}{l}55 \\
56\end{array}$ & $\begin{array}{l}55^{2} \\
53^{2}\end{array}$ & $\begin{array}{l}67 \\
62\end{array}$ & $\begin{array}{l}0,145^{2} \\
0,149^{1}\end{array}$ \\
\hline 12 & $\begin{array}{l}\text { OD * } \\
\text { OE }\end{array}$ & $\begin{array}{l}0,72^{2} \\
0,90\end{array}$ & $\begin{array}{l}1,19^{1} \\
1,81\end{array}$ & $\begin{array}{l}1,66 \\
2,00\end{array}$ & $\begin{array}{l}1,12^{2} \\
1,76\end{array}$ & $\begin{array}{l}0,66^{2} \\
1,00\end{array}$ & $\begin{array}{l}0,94^{2} \\
1,84\end{array}$ & $\begin{array}{l}58^{1} \\
21\end{array}$ & $\begin{array}{l}96 \\
96\end{array}$ & $\begin{array}{l}98 \\
99\end{array}$ & $\begin{array}{r}90 \\
108\end{array}$ & $\begin{array}{l}119 \\
117\end{array}$ & $\begin{array}{l}0,250 \\
0,292\end{array}$ \\
\hline 13 & $\begin{array}{l}\text { OD } \\
\text { OE }\end{array}$ & $\begin{array}{l}0,90 \\
0,92\end{array}$ & $\begin{array}{l}2,46 \\
1,80\end{array}$ & $\begin{array}{l}2,74 \\
1,96\end{array}$ & $\begin{array}{l}1,84 \\
1,61\end{array}$ & $\begin{array}{l}1,74 \\
0,96^{1}\end{array}$ & $\begin{array}{l}4,69 \\
2,53\end{array}$ & $\begin{array}{l}14 \\
29\end{array}$ & $\begin{array}{l}55 \\
55\end{array}$ & $\begin{array}{l}56 \\
56\end{array}$ & $\begin{array}{l}49^{2} \\
49^{2}\end{array}$ & $\begin{array}{l}78 \\
71\end{array}$ & $\begin{array}{l}0,133^{2} \\
0,133^{2}\end{array}$ \\
\hline
\end{tabular}

de oxigênio à circulação da retina, levando à perda parcial de fibras da retina.

\section{ABSTRACT}

Purpose: To evaluate findings of ophthalmologic examinations in cardiac transplant recipients, searching especially for changes in the retinal nerve fiber layer by means of Scanning Laser Polarimetry. Methods: Fifteen cardiac transplant recipients were examined from September 2003 to July 2004. All of them underwent ophthalmologic examination, which consisted of visual acuity (VA), biomicroscopy, tonometry and fundoscopy. Fiber layer analyzer - GDx - examination was performed in eleven patients. Twelve patients were men. The mean age was $55.0 \pm 13.5$ years. The follow-up since transplantation lasted from 3 to 74 months; mean value $29.7 \pm$ 20.8 months. Results: VA with best correction in all patients attained at least 20/40. Subcapsular posterior cataract was seen in one patient; another presented corneal nubeculae secondary to herpes zoster. In one case a scar suggesting retinocoroiditis was seen at fundoscopy. Biomicroscopic and the fundoscopic findings were expected because of immunosuppressive treatment, following transplantation. GDx examination disclosed loss of fibers in the superior retinal fiber layer in 12 of the 22 examined eyes. Conclusion: These results support the hypothesis that reduction of oxygen inflow in retinal circulation before or during heart transplantation could lead to loss of fibers in the retinal nerve fiber layer.
Keywords: GDx; Heart transplantation; Eye manifestations/etiology; Nerve fibers; Diagnostic techniques, ophthalmological

\section{REFERÊNCIAS}

1. Magalhães HM, Manrique R, Dinkhuysen J, Pavanello R, Magalhães Filho HM. Transplante cardíaco. In: Porto CC, Porto AL, Organizadores. Doenças do coração: prevenção e tratamento. $2^{\underline{a}}$ ed. Rio de Janeiro: Guanabara Koogan; 2005. p.1030-1033.

2. Ng P, McCluskey P, McCaughan G, Glanville A, MacDonald P, Keogh A. Ocular complications of heart, lung, and liver transplantation. Br J Ophthalmol. 1998;82(4):423-8.

3. Friberg TR, Eller AW. Serous retinal detachment resembling central serous chorioretinopathy following organ transplantation. Graefes Arch Clin Exp Ophthalmol. 1990;228(4):305-9.

4. Allinson RW, Limstrom SA, Sethi GK, Copeland JG. Central retinal vein occlusion after heart-lung transplantation. Ann Ophthalmol. 1993;25(2):58-63.

5. Chou PI, Lee H, Lee FY. Cytomegalovirus retinitis after heart transplant: a case report. Zhonghua Yi Xue Za Zhi (Taipei). 1996;57(4):310-3.

6. Yee RW, Sigler SC, Lawton AW, Alderson GL, Trinkle JK, Lum CT. Apparent cytomegalovirus epithelial keratitis in a cardiac transplant recipient. Transplantation. 1991;51(5):1040-3.

7. Quinlan MF, Salmon JF. Ophthalmic complications after heart transplantation. J Heart Lung Transplant. 1993;12(2):252-5.

8. Emmrich P, Hahn J, Ogunlade V, Geiger K, Schober R, Mohr FW. [Neuropathological findings after cardiac surgery - retrospective study over 6 years]. Z Kardiol. 2003;92(11):925-37. German.

9. Wapner FJ, Leib ML, Drusin R, Rose E, Srinivasan BD. Ocular complications associated with cardiac transplantation. Trans Am Ophthalmol Soc. 1992;90:171-8; discussion 178-81.

10. Benedict RH. Cognitive function after open-heart surgery: are postoperative neuropsychological deficits caused by cardiopulmonary bypass? Neuropsychol Rev. 1994;4(3):223-55.

11. Luo X, Lambrou GN, Sahel JA, Hicks D. Hypoglycemia induces general neuronal death, whereas hypoxia and glutamate transport blockade lead to selective retinal ganglion cell death in vitro. Invest Ophthalmol Vis Sci. 2001;42(11):2695-705. 